\title{
El Perú en el Tratado de Asociación Transpacífico (TPP): beneficios y mitos
}

\author{
José Luis Castillo Mezarina*
}

\section{RESUMEN}

El Tratado de Asociación Transpacífico (TPP) es el primer acuerdo comercial mega-regional del siglo XXI. Está conformado por doce economías de tres continentes, todas miembros del Foro de Cooperación Económica Asia-Pacífico (APEC).

La participación del Perú en este excepcional Tratado implica ganar cinco nuevos mercados en un solo acuerdo comercial: Australia, Brunéi Darussalam, Nueva Zelanda, Malasia y Vietnam. Se estima el incremento potencial de las exportaciones no tradicionales peruanas a dichos mercados en US\$ 2250 millones con la entrada en vigencia del TPP. El país gana también por la mejora en las condiciones de acceso y reglas pactadas en los acuerdos bilaterales previos.

Una de las mayores ganancias derivadas del TPP para las economías en desarrollo como el Perú es el acceso a las cadenas globales de valor, como resultado de la aplicación del mecanismo conocido como «acumulación de origen». Este mecanismo beneficiará particularmente a las pyme, pues podrán exportar insumos que se engarcen en una cadena de valor en el bloque TPP.

Con relación a los mitos sobre impactos negativos del TPP, específicamente sobre una supuesta elevación de precios de medicamentos, supuestas pérdidas millonarias en arbitrajes inversionista-Estado y supuestas restricciones a las libertades en internet, no es cierto que el Tratado genere efectos adversos en estas áreas. Los mismos mitos se difundieron durante la negociación del Acuerdo de Promoción Comercial Perú-Estados Unidos de América (EE. UU.) y nada de ello ocurrió en la práctica.

Palabras clave: negociaciones, libre, comercio, TPP, transpacífico, tlc, propiedad, intelectual, medicamentos, patentes, inversión, acceso, mercados, cadenas de valor.

\footnotetext{
* Ministerio de Comercio Exterior y Turismo, Lima - Perú. Licenciado en Economía por la Universidad del Pacífico. Correo electrónico: jlcastillo@mincetur.gob.pe
} 


\section{Peru in the Trans-Pacific Partnership (TPP): Benefits and Myths}

\section{Abstract}

The Trans-Pacific Partnership Agreement (TPP) is the first mega-regional trade agreement of the XXI century. It is composed of twelve economies of three continents, all members of the Asia-Pacific Economic Cooperation (APEC).

Peru's participation in this exceptional agreement means winning five new markets in a single trade agreement: Australia, Brunei Darussalam, New Zealand, Malaysia and Vietnam. The potential increase in non-traditional Peruvian exports to those markets by the entry into force of the TPP Agreement is estimated in US\$2,250 million. The country also gains by improving in TPP the conditions of access and trade rules of previous bilateral agreements.

One of the biggest gains from TPP for developing economies like Peru is the access to global value chains, as a result of the application of a mechanism known as «origin accumulation». This mechanism will particularly benefit SMEs, as inputs they export can be introduced in a value chain of the TPP trading bloc.

Regarding myths about negative impacts of the TPP, specifically about a supposed rise in prices of medicines, alleged millionaire losses in Investor-State Dispute Settlement arbitrations, and alleged restrictions on freedoms in the Internet, it is not true that the TPP Agreement generates adverse effects in these areas The same myths were spread during of the US-Peru Trade Promotion Agreement negotiations, and none of it happened in reality.

Key words: Negotiations, Free, Trade, TPP, Trans-Pacific, FTA, Intellectual, Property, Pharmaceuticals, Patents, Investment, Market, Access, Value Chains. 


\section{El TPP en el contexto de las negociaciones comerciales del Perú}

El Tratado de Asociación Transpacífico es un acuerdo comercial plurilateral, suscrito por doce economías de tres continentes: América, Asia y Oceanía, todas miembros del Foro de Cooperación Económica Asia-Pacífico (APEC)ํㅡㄹ ${ }^{1}$ El TPP está conformado por Australia, Brunei Darussalam, Canadá, Chile, Estados Unidos, Japón, México, Malasia, Nueva Zelanda, Singapur, Perú y Vietnam.

Gráfico 1. Países miembros del TPP

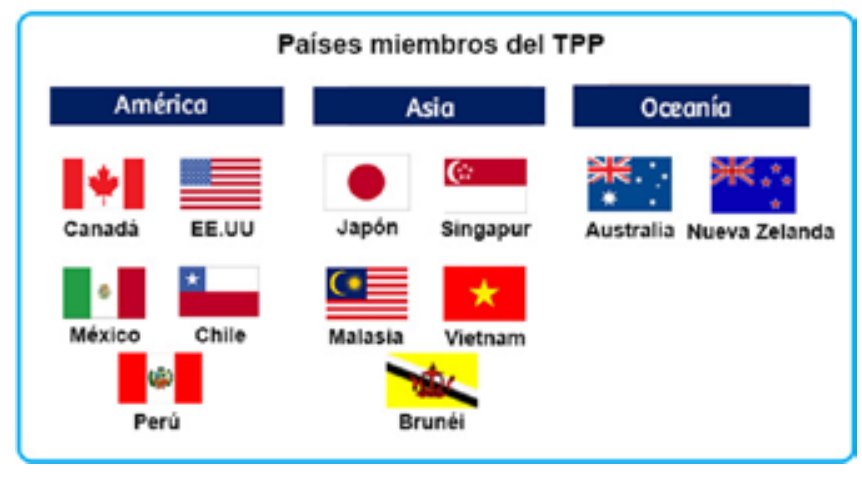

Fuente: Mincetur. Elaboración: Mincetur.

La iniciativa busca conformar un área de libre comercio que abarque el comercio de bienes, servicios, las inversiones y la movilidad de personas de negocios, así como propiedad intelectual, asuntos laborales y medioambientales, entre otros. Tiene como objetivo construir un acuerdo inclusivo y de alta calidad, que sea soporte para el crecimiento económico, el desarrollo y la generación de empleo de sus países miembros $^{2}$. Asimismo, busca convertirse en la base para la futura Área de Libre Comercio del Asia Pacífico (FTAAP).

El TPP debe ser contextualizado en el marco del proceso de apertura del Perú al comercio internacional y la posterior negociación de acuerdos de libre comercio bilaterales. El Perú suma más de 25 años de políticas comerciales consistentes, desde inicios de la década de 1990, con la apertura a los mercados internacionales. Luego, esta política fue complementada con la negociación de acuerdos comerciales bilaterales, siendo el máximo referente el Acuerdo de Promoción Comercial (APC) entre los Estados Unidos y el Perú, vigente desde 2009.

\footnotetext{
APEC está conformado por 21 economías miembros. El Perú forma parte de APEC desde 1998.

2 www.acuerdoscomerciales.gob.pe
} 
El Ministerio de Comercio Exterior y Turismo (Mincetur) cataloga al TPP como un paso lógico y consistente en la dirección correcta: la coronación de un proceso de apertura iniciado por el país hace casi ya un cuarto de siglo (Mincetur, 2015, pp. 3-11).

\section{Orígenes del TPP}

El primer antecedente histórico del Tratado de Asociación Transpacífico es el anuncio de inicio de negociaciones de un acuerdo trilateral estratégico de asociación económica entre Chile, Nueva Zelanda y Singapur, durante la cumbre de líderes del Foro de Cooperación Económica Asia-Pacífico (APEC) en Los Cabos, México, en octubre de $2002^{3}$. Las negociaciones del acuerdo, conocido como P3, iniciaron en marzo de 2003. Luego, en 2005, Brunei Darussalam se incorpora a las negociaciones y el acuerdo se renombra como Acuerdo Estratégico Transpacífico de Asociación Económica (P4), suscrito en 2005 y vigente para sus miembros desde 2006.

Un dato relevante es que el gobierno de Nueva Zelanda se dirigió al Mincetur para invitar al Perú a adherirse al recientemente puesto en vigencia Acuerdo P4. Sin embargo, luego de analizar cada uno de los capítulos, el Perú decidió no aceptar la invitación de Nueva Zelanda, debido a que se trataba de una adhesión a un acuerdo vigente y el país no había podido participar en la definición de las reglas u obligaciones del Acuerdo P4 durante la etapa de negociación.

En febrero de 2008, Estados Unidos anunció el inicio de negociaciones sobre servicios financieros e inversión con los miembros del P4, así como su intención de iniciar un proceso de exploración detallado, para determinar si debía involucrarse en negociaciones para un acuerdo de libre comercio completo con el P4 (Executive Office of the President of the United States, 2008). Luego, el 22 de setiembre de 2008, la Representante de Comercio de los Estados Unidos, Susan Schwab, anunció que su país iniciaría negociaciones con los miembros del P4 para conformar el Tratado de Asociación Transpacífico o TPP.

En noviembre de 2008, durante la cumbre de líderes de APEC en Lima, Australia, el Perú y Vietnam anunciaron su intención de unirse a las negociaciones del TPP. Este anuncio fue formalizado el 1 de diciembre de 2008, mediante carta enviada por el Ministerio de Comercio Exterior y Turismo del Perú al Ministerio de Relaciones Exteriores y Comercio de Nueva Zelanda. El 10 de febrero de 2009 los miembros del TPP aceptaron formalmente la participación del Perú en el proceso que estaba

\footnotetext{
3 Véase: Sistema de Información sobre Comercio Exterior (SICE) de la Organización de Estados Americanos (OEA). Disponible en: <http://www.sice.oas.org/TPD/CHL_Asia/CHL_Asia_s.ASP>.
} 
por iniciarse. Las negociaciones comenzaron el 15 marzo de 2010 en Melbourne, Australia. Posteriormente, durante la tercera ronda, en octubre de 2010, Malasia ingresó a las negociaciones del TPP y Vietnam formalizó su participación como miembro pleno del grupo, pues hasta ese momento tenía calidad de observador. Por su parte, Canadá y México lograron incorporarse en octubre de 2012 y el último país en adherirse al proceso fue Japón, en mayo de 2013.

\section{Gráfico 2. Línea de tiempo del TPP}

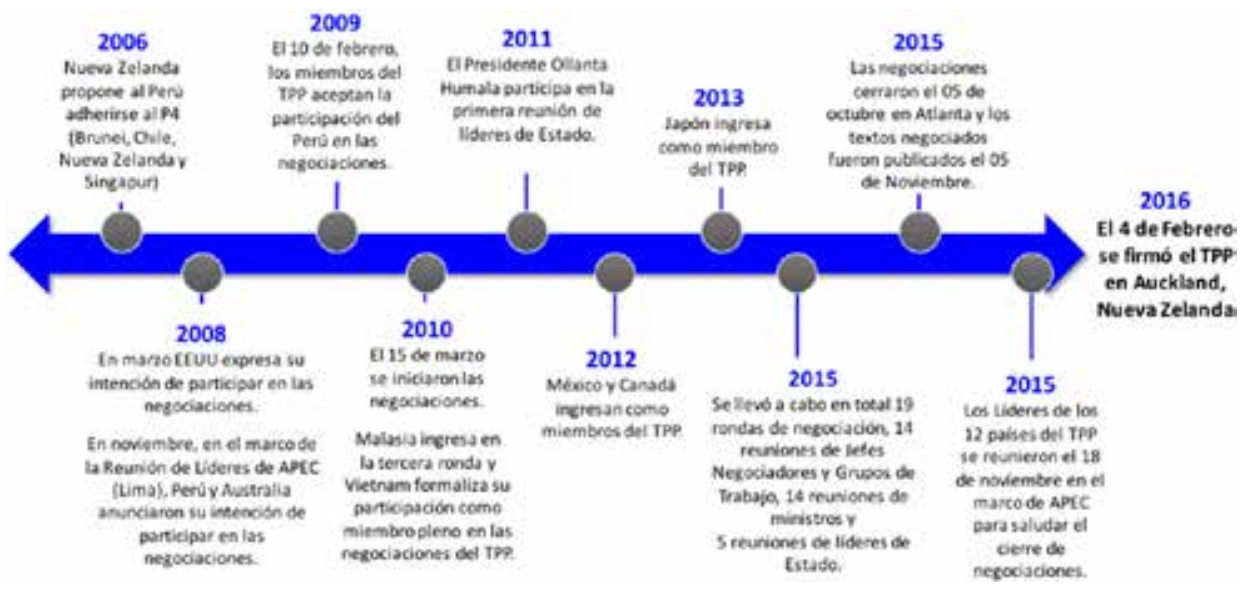

Fuente y elaboración: Mincetur.

Entre marzo de 2010 y octubre de 2015 los países miembros del TPP realizaron diecinueve rondas de negociación, catorce reuniones de jefes negociadores y grupos técnicos, catorce reuniones de ministros de comercio y cinco reuniones de líderes de Estado.

El cierre de negociaciones se alcanzó el 5 de octubre de 2015 en Atlanta, Estados Unidos. Luego, atendiendo al gran interés de la población por conocer los textos negociados, el 5 de noviembre de 2015, un mes después del cierre de negociaciones, los doce países miembros publicaron en simultáneo el íntegro de los textos del TPP, a pesar de que aún quedaba pendiente concluir el proceso de revisión y coherencia legal de los textos.

La firma del TPP en sus tres idiomas oficiales, inglés, español y francés —un hecho histórico sin precedentes por la magnitud de las economías que lo conforman y la profundidad de los compromisos alcanzados - se produjo el 4 de febrero en la ciudad de Auckland, Nueva Zelanda. Dicho país es el depositario del Tratado. 


\section{El bloque comercial más grande a escala mundial}

El TPP será el bloque más grande a escala mundial en términos comerciales y económicos, por encima incluso de la Unión Europea (UE), pues en conjunto los doce países miembros constituyen un mercado ampliado de 818 millones de habitantes —casi el 12\% de la población mundial— con un PBI per cápita promedio de US\$ 28448 . Conjuntamente, los países del TPP representan cerca del $40 \%$ del producto bruto mundial, el 25\% de las exportaciones y el 27\% de las importaciones globales 4 .

En el siguiente cuadro se detalla los datos de población y PBI per cápita para cada uno de los miembros del TPP:

\section{Cuadro 1. TPP: población y PBI per cápita}

\begin{tabular}{|clr|}
\hline \multicolumn{3}{|c|}{$\begin{array}{c}\text { Población de países miembros del TPP } \\
(2015)\end{array}$} \\
\hline Puesto & País & Millones \\
\hline & & 321,6 \\
2 & Estados Unidos $\left(^{*}\right)$ & 127,0 \\
3 & México $\left(^{*}\right)$ & 126,9 \\
4 & Japón & 91,7 \\
5 & Vietnam $\left(^{*}\right)$ & 35,8 \\
6 & Canadá & 31,9 \\
7 & Perú $\left(^{*}\right)$ & 31,0 \\
8 & Malasia & 24,0 \\
9 & Chstralia & 18,0 \\
10 & Singapur $\left(^{*}\right)$ & 5,5 \\
11 & Nueva Zelandia & 4,6 \\
12 & Brunéi $\left(^{*}\right)$ & 0,4 \\
\hline & Total del TPP & 818,4 \\
\hline
\end{tabular}

\begin{tabular}{|c|c|c|}
\hline \multicolumn{3}{|c|}{$\begin{array}{c}\text { PBI per cápita de países miembros } \\
\text { del TPP }(2015)\end{array}$} \\
\hline Puesto & País & $\begin{array}{c}\text { US\$ / } \\
\text { habitante }\end{array}$ \\
\hline 1 & Estados Unidos $\left(^{*}\right)$ & 55805 \\
\hline 2 & Singapur $\left(^{*}\right)$ & 52888 \\
\hline 3 & Australia & 50962 \\
\hline 4 & Canadá $(*)$ & 43332 \\
\hline 5 & Nueva Zelanda & 37045 \\
\hline 6 & Japón $\left(^{*}\right)$ & 32486 \\
\hline 7 & Brunéi & 28837 \\
\hline 8 & Chile & 13341 \\
\hline 9 & Malasia & 9557 \\
\hline 10 & México $\left(^{*}\right)$ & 9009 \\
\hline 11 & Perú & 6021 \\
\hline 12 & Vietnam $\left(^{*}\right)$ & 2088 \\
\hline \multicolumn{2}{|c|}{ Promedio del TPP } & 28448 \\
\hline
\end{tabular}

(*) Datos estimados para 2015.

Fuente: FMI y World Economic Outlook. Elaboración: Mincetur.

El Perú se ubica en la mitad de la tabla de los países del TPP en cuanto al tamaño de su población. Sin embargo, en términos del PBI per cápita, se ubica casi el final de la tabla, solamente superando a Vietnam.

Si bien la ubicación del Perú al final de la tabla del PBI per cápita puede ser percibida como un riesgo en términos de una relación asimétrica con los demás socios del TPP, este hecho no representa más que una oportunidad, ya que el Perú se está asociando con economías de primer nivel y con un gran poder adquisitivo. Ello significa que los

4 El bloque europeo suma poco más de 500 millones de habitantes y alrededor del 25\% del PBI mundial. 
empresarios peruanos podrán acceder a un mercado ampliado y ofrecer sus productos a economías con un promedio de ingreso per cápita superior a los US\$28 000 por habitante al año 2015.

\section{La negociación del TPP}

La negociación del TPP se organizó en 20 grupos de negociación, que tuvieron a su cargo los 30 capítulos que componen el texto final suscrito por sus miembros (véase cuadro 2). Los temas tratados son diversos, pues abarcan desde el comercio de bienes y servicios, hasta inversión, contratación pública y propiedad intelectual.

$\mathrm{Al}$ ser el TPP el primer gran acuerdo de libre comercio del siglo XXI —a la vez que el primer acuerdo mega regional que incorpora economías desarrolladas y economías en vías de desarrollo- sus miembros decidieron abordar no solamente los temas clásicos de los acuerdos comerciales, sino buscaron incorporar también disposiciones y temas innovadores en la negociación, tales como los capítulos sobre Empresas de Propiedad del Estado y Monopolios Designados, Competitividad y Facilitación de Negocios, Coherencia Regulatoria, Pequeñas y Medianas Empresas (pymes) y Desarrollo. Estos son temas que no han sido abordados en acuerdos comerciales previos.

\section{Cuadro 2. Capítulos del TPP}

\begin{tabular}{|c|l|c|l|}
\hline 1 & Disposiciones iniciales y definiciones & 15 & Contratación pública \\
2 & generales & 16 & Competencia \\
3 & Trato nacional y acceso de mercancías & 17 & Empresas de propiedad del Estado y \\
& al mercado & & monopolios designados \\
& Reglas de origen y procedimientos & 18 & Propiedad intelectual \\
4 & relacionados con el origen & 19 & Laboral \\
5 & Textiles y confecciones & 20 & Medio ambiente \\
& Administración aduanera y facilitación & 21 & Cooperación y desarrollo de capacidades \\
6 & del comercio & 22 & Competitividad y facilitación de \\
7 & Mefensa comercial & & negocios \\
8 & Obstáculos técnicos al comercio & 23 & Desarrollo \\
9 & Inversión & 24 & Pequeñas y medianas empresas - pymes \\
10 & Comercio transfronterizo de servicios & 25 & Transparencia y anticorrucpión \\
11 & Servicios financieros & 26 & Disposiciones admnistrativas e \\
12 & Entrada temporal para personas de & 27 & institucionales \\
& negocios & 28 & Solución de controversias \\
13 & Telecomunicaciones & 29 & Excepciones y disposiciones generales \\
14 & Comercio electrónico & 30 & Disposiciones finales \\
\hline
\end{tabular}

Fuente y elaboración: Mincetur. 


\section{Relación con acuerdos preexistentes}

Bagwati (1995) calificó como «spaghetti bowl effect» al efecto que genera la maraña de acuerdos comerciales que se superponen unos con otros, «refiriéndose a la manera en la que productos semifinalizados y partes utilizan diferentes redes de TLC aprovechando la diferenciación arancelaria en un esfuerzo para exportar productos finales a los países consumidores al precio más bajo».

A la fecha de elaboración de este artículo, el Perú cuenta con 18 acuerdos comerciales vigentes, tres acuerdos suscritos no vigentes y cinco en negociación. El TPP se sumará a esta red de acuerdos comerciales, en la que evidentemente se genera una superposición de acuerdos.

\section{Gráfico 3. Red de Acuerdos comerciales del Perú}

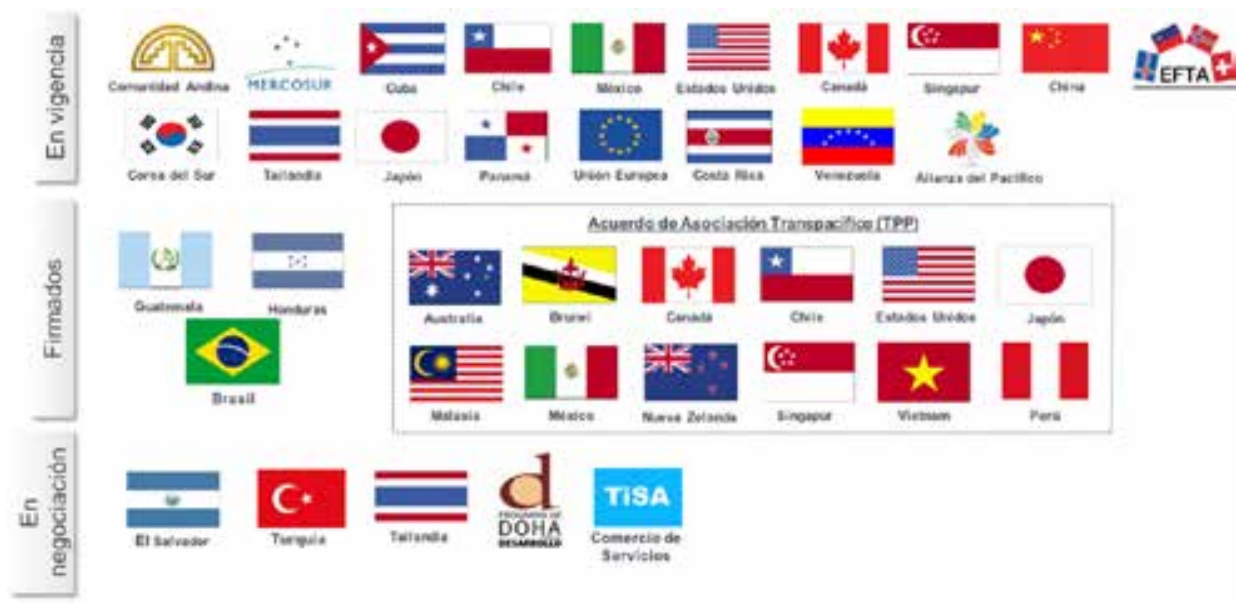

Fuente y elaboración: Mincetur.

Esta superposición puede ser ejemplificada en la relación comercial bilateral entre Perú y Chile de la siguiente manera: el Perú cuenta con un acuerdo de libre comercio bilateral con Chile vigente desde 2009. Asimismo, el Perú ha suscrito el protocolo comercial de la Alianza del Pacífico, junto con Chile, Colombia y México. En adición, tanto Chile como el Perú forman parte de los doce países miembros del TPP. Por tanto, cuando todos estos acuerdos estén vigentes habrá tres instrumentos internacionales que rijan las relaciones comerciales entre el Perú y Chile.

Luego de constatar esta situación, las preguntas lógicas son las siguientes: ¿es esto una política coherente? ¿Qué acuerdo prevalecerá? Las respuestas son sencillas: (i) sí es una política coherente porque permite brindar al operador de comercio exterior más instrumentos de dónde elegir; y (ii) en ningún caso un acuerdo comercial prevalecerá 
sobre otro. Todos los acuerdos seguirán vigentes y coexistirán en el tiempo. No se derogará acuerdo previo alguno entre los miembros del TPP una vez que entre en vigencia el Tratado5.

En el caso peruano, el país cuenta con acuerdos bilaterales con seis economías que también participan en el TPP: Canadá, Chile, Estados Unidos, Japón, México y Singapur. Estos acuerdos continuarán vigentes y coexistirán con el TPP. Serán los usuarios de los acuerdos -importadores, exportadores e inversionistas - los que elijan cuál de los tratados utilizar, ya sea el acuerdo bilateral que corresponda o el TPP, dependiendo de cuál es el instrumento más ventajoso para sus intereses.

Por ejemplo, si un acuerdo bilateral excluye la línea arancelaria de interés para el exportador, pero esa misma línea arancelaria recibe una preferencia en el marco del TPP, evidentemente el exportador elegirá el TPP en lugar del acuerdo bilateral. Por el contrario, si el acuerdo bilateral ofrece una mejor preferencia arancelaria para el producto debido, por ejemplo, a que el cronograma de desgravación arancelaria de dicho acuerdo está mucho más avanzado que el cronograma de desgravación arancelaria del TPP, entonces el exportador elegirá exportar utilizando el acuerdo bilateral.

\section{Beneficios del TPP para el Perú}

De acuerdo con un reciente estudio publicado por el Banco Mundial, que evalúa los impactos macroeconómicos del TPP, se estima que como resultado de la aplicación del Tratado el crecimiento económico de las economías del TPP proyectado al año 2030 se ubicará en un rango entre $0,4 \%$ y $10 \%$, y será de $1,1 \%$ sobre una base ponderada por PBI (World Bank, 2016, p. 227). Si dicho crecimiento es el estimado para una región que representa cerca del $40 \%$ del PBI global, es lógico anticipar que el PBI mundial crecerá como resultado de la aplicación del TPP.

En el caso peruano, de acuerdo con la investigación del Banco Mundial, se estima que el PBI crezca en el orden de $2 \%$ a $4 \%$ y se espera que las exportaciones se incrementen entre $10 \%$ y $15 \%$ para el año 2030 .

Asimismo, otro estudio sobre elTPP, realizado por el Peterson Institute for International Economics, estima que para el año 2025 el TPP generará para el Perú 6 mil millones de dólares de ingreso real anual y para 2030 se espera US\$ 11 mil millones de dólares o 2,6\% del PBI. En cuanto al comportamiento de las exportaciones, se espera que estas se incrementen en 14 mil millones de dólares o 10,3\% para el año 2030

\footnotetext{
5 Durante la negociación del TPP se tomó especial cuidado en evitar inconsistencias entre el nuevo acuerdo y los instrumentos previos que rigen las relaciones comerciales entre sus miembros.
} 
(Petri y Plummer, 2016, pp. 20-21). Con la entrada en vigencia del TPP, en adición a las ganancias macroeconómicas, el Perú se encontrará en una posición estratégicamente más competitiva, pues existen múltiples beneficios en términos de acceso a mercados para bienes y servicios, reglas de origen, medidas sanitarias y fitosanitarias, inversiones, medioambiente, entre otros.

El Perú gana con el TPP cinco nuevos mercados en un solo acuerdo comercial: Australia, Brunéi Darussalam, Malasia, Nueva Zelanda y Vietnam. Se estima que el impacto de esta nueva asociación comercial incidirá, en primer lugar, en el volumen de las exportaciones no tradicionales peruanas; en especial en sectores como agroindustria, pesca, confecciones de algodón y alpaca, y manufacturas diversas. Dado que la participación del Perú en el total de compras de dichos productos por parte de los cinco países mencionados no supera el $1 \%$, se sabe que el potencial para incrementar las exportaciones a dichos mercados es enorme. El Mincetur ha estimado el impacto positivo del TPP en $25 \%$ del total las exportaciones no tradicionales peruanas.

Adicionalmente, con el TPP el Perú mejorará las condiciones de acceso a mercados obtenidas en los tratados bilaterales vigentes, especialmente con Japón y Canadá. En el primer caso, el Acuerdo de Asociación Económica entre Japón y Perú mantiene un total de 1062 líneas arancelarias excluidas, que no gozan de preferencia arancelaria alguna. A diferencia de este acuerdo bilateral, en el TPP Japón solamente mantiene 133 líneas arancelarias excluidas ${ }^{6}$. En consecuencia, con el TPP, el Perú está mejorando en hasta 10 puntos porcentuales el número de productos beneficiados con Japón, entre los cuales se encuentra el atún congelado, caballa congelada, trucha, leche, galletas, preparaciones de chocolate y preparaciones para alimentación de animales, entre otros. En el caso de Canadá, las ganancias con respecto al Acuerdo de Libre Comercio bilateral se concentran en el sector agropecuario, especialmente en lácteos y aves de corral.

El TPP no solamente es importante para el Perú por los nuevos mercados que abrirá, sino en especial porque contempla el mecanismo denominado "acumulación de origen» que facilitará y promoverá la inserción de las empresas peruanas en cadenas globales de valor. La acumulación de origen del Tratado permite a las empresas de los países miembros abastecerse y ser abastecedoras de insumos desde y hacia los doce países que conforman la región TPP. Con ello, las posibilidades para que las empresas peruanas obtengan beneficios tangibles del Tratado se incrementan exponencialmente.

\footnotetext{
6 Se ha utilizado el Sistema Armonizado de 2007 (HS 2007) para comparar el acuerdo bilateral y el TPP. Cabe señalar que las listas finales del TPP han sido publicadas en HS 2012.
} 
El mecanismo de acumulación de origen que contempla el TPP permitirá que las pyme peruanas puedan insertarse de manera efectiva en las cadenas regionales y globales de valor. Gracias a este mecanismo, una pyme peruana podrá adquirir los mejores insumos a los precios más competitivos en cualquiera de los otros once países miembros del TPP e importarlos al Perú con preferencias arancelarias. Luego, dicha pyme podrá producir un bien final con dichos insumos y exportarlo a cualquier otro país del bloque con las preferencias arancelarias negociadas en el Tratado.

Del mismo modo, una pyme peruana también podrá abastecer de insumos a empresas de otro país del TPP, para que dichas empresas elaboren un bien final que a su vez se exportará a un tercer país del TPP. De esta manera, se fomenta la integración económica y la consolidación de la diversificación productiva.

\section{Gráfico 4. El TPP y las Cadenas Globales de Valor}

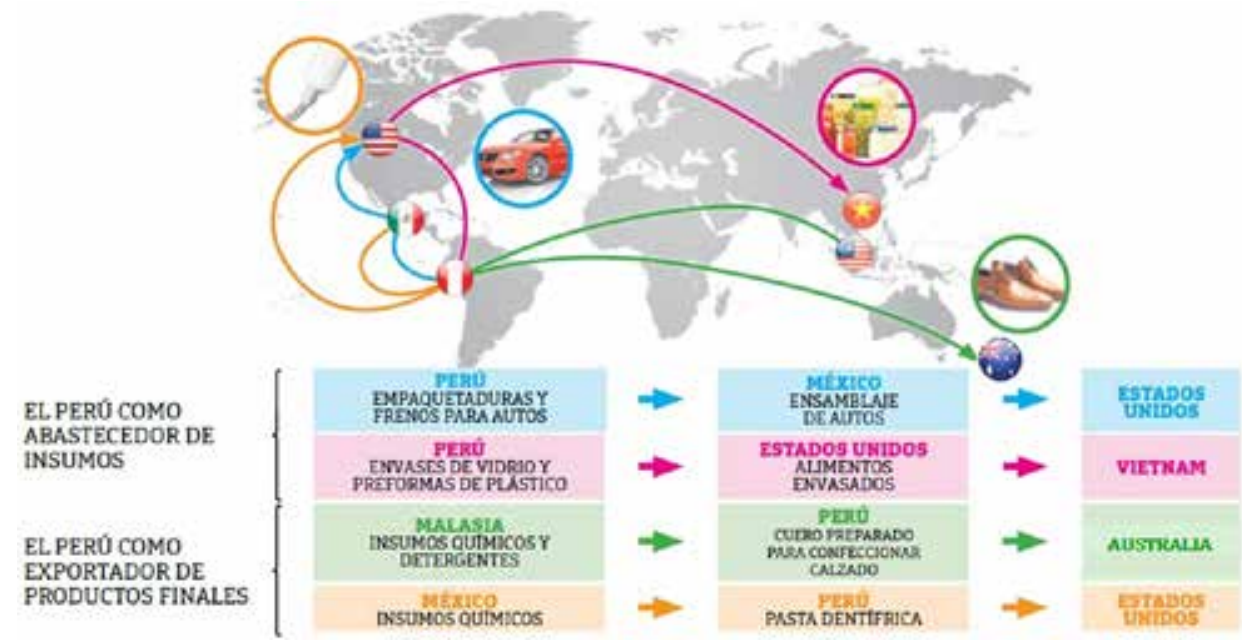

Fuente: Mincetur. Elaboración: Mincetur

A modo de ejemplo, una PYME ubicada en el Perú podría producir autopartes, como muelles o empaquetaduras para motores de automóvil; luego, esas partes y piezas se pueden exportar con preferencias arancelarias del TPP a México, país ensamblador de automóviles, y posteriormente dicho país puede a su vez exportar el automóvil a los Estados Unidos con las preferencias arancelarias del TPP. Esta cadena de preferencias no sería posible en el marco de los acuerdos bilaterales existentes, a pesar de que Estados Unidos, México y el Perú cuentan con acuerdos bilaterales entre sí.

Otro ejemplo de las bondades de la acumulación del origen sería el siguiente: el Perú produce y exporta envases de vidrio a los Estados Unidos con las preferencias 
arancelarias del TPP, para que luego dicho país elabore alimentos procesados utilizando los envases de vidrio producidos en el Perú, y proceda luego a exportar el producto final a Japón con las preferencias del Tratado.

Es preciso destacar que el TPP representa la primera vez que un acuerdo de libre comercio incorpora un capítulo específico en beneficio de las PYMEs e incluye disposiciones para insertarlas efectivamente en el gran juego del comercio internacional, a través de las cadenas globales de valor.

Asimismo, el TPP será el primer acuerdo comercial en el que sus países miembros destacan la importancia del crecimiento inclusivo, la igualdad de género y la educación. Estos temas fueron incluidos en la negociación a propuesta del Perú y se plasmaron finalmente en un capítulo específico denominado "Desarrollo». Este es un capitulo novedoso a nivel mundial, que resalta la importancia de que la política comercial sea acompañada de políticas de inclusión social que logren aprovechar de mejor manera las oportunidades que surgen del acuerdo comercial.

En materia sanitaria y fitosanitaria, tema de vital importancia para un país agroexportador como el Perú, el TPP cuenta con mecanismos eficientes y ágiles para evitar demoras injustificadas e indebidas en el ingreso de estos productos a los mercados de los socios comerciales en el TPP. Entre ellos, destaca la sujeción del capítulo al mecanismo de solución de controversias del Tratado. En efecto, mientras que en el APC Perú - Estados Unidos o el EPA Japón - Perú el capítulo sobre medidas sanitarias y fitosanitarias no está sujeto a los mecanismos de solución de controversias previstos en los respectivos Acuerdos, en el TPP sí lo está. De esta manera, el Estado Peruano contará ahora con las herramientas necesarias para abrir un caso que dirima una eventual controversia internacional con los Estados Unidos o Japón, ante un supuesto de incumplimiento de las obligaciones del capítulo sobre medidas sanitarias y fitosanitarias. Ello, en el contexto de los acuerdos bilaterales entre el Perú y los Estados Unidos o Japón, no es posible.

El TPP permitirá también que los empresarios peruanos participen en los procesos de compras públicas de bienes y servicios de los otros miembros del TPP, en mejores condiciones que terceros países. Ello debido a que el Tratado incluye la obligación de otorgar "Trato Nacional», lo cual implica que las entidades contratantes de los Estados miembros deben otorgar a los proveedores provenientes de países del TPP, así como a los bienes y servicios provenientes del TPP, un trato no menos favorable que el otorgado a sus propios proveedores, bienes y servicios. El sistema peruano de compras públicas ya está abierto al mundo y, en consecuencia, no existe discriminación entre proveedores peruanos o extranjeros. El TPP permitirá que el resto de países miembros abra también sus mercados de compras públicas a los proveedores, bienes y servicios peruanos en igualdad de condiciones. 
En el ámbito del comercio de servicios, las transacciones internacionales presentan características peculiares. Los servicios son intangibles, por lo que su ingreso a los países no se produce a través de las aduanas. Dadas estas características, las restricciones que imponen los países a este tipo de transacciones se encuentran en la regulación nacional, la misma que podría ser discriminatoria o imponer límites cuantitativos que impidan o limiten el comercio de servicios ${ }^{7}$. El TPP cuenta con disposiciones que permitirán a los exportadores de servicios peruanos proveer servicios a los consumidores dentro del bloque con la debida certeza jurídica y evitando restricciones. En este sector destacan los exportadores peruanos de servicios call-centers, consultoría, procesamiento de datos, servicios profesionales, creación de software, diseño de modas, entre otros.

Con respecto al flujo de inversiones, el TPP permitirá atraer un mayor flujo de Inversión Extranjera Directa (IED) desde los países miembros del TPP al Perú. Ello debido a que, en la medida que el TPP representa para los inversionistas de dichos países una garantía de respeto y transparencia para sus inversiones, el Perú podrá potenciar sus atractivos para los inversionistas en sectores como el minero, pesquero, agroexportador, e industrial. Además, si bien el Perú es un país importador neto de capital, no debe olvidarse la perspectiva y proyección de exportar capital, pues cada vez más las empresas peruanas se están internacionalizando; por ello, es de vital importancia otorgar también los instrumentos de certidumbre y protección de la inversión peruana en los países del TPP.

El capítulo de Entrada Temporal de Personas de Negocios permitirá evitar discriminación en contra de los peruanos en el otorgamiento autorizaciones de ingreso y permanencia para personas de negocios. El compromiso sobre Autorización de Entrada Temporal del referido capítulo brinda certeza sobre las categorías de personas de negocios que cada país del TPP autoriza en su territorio. Con ello se beneficiará a profesionales y técnicos, tales como chefs, técnicos en ingeniería, enfermeros, además de empresarios e inversionistas peruanos.

El capítulo de medioambiente brinda aportes a la solución de problemas ambientales globales, desde la perspectiva del comercio. Se ha incluido disposiciones sobre la conservación de especies en peligro de extinción, transición hacia economías de baja emisión, subsidios a la pesca, entre otros. Para el Perú la obligación más importante en el capítulo de medioambiente del TPP es el artículo sobre comercio y biodiversidad, que fue incluido en el TPP a propuesta del Perú. Dicho artículo reconoce

\footnotetext{
7 Un ejemplo de una medida que restringe el comercio de servicios sería la existencia de una ley o norma de menor rango que disponga que las aplicaciones de teléfonos móviles o deben ser producidas en el país. Con ello, se impide que desarrolladores de otros países presten el servicio de desarrollo de aplicativos móviles.
} 
la importancia de la conservación y el uso sostenible de la biodiversidad a través del comercio. Cabe recordar que el Perú es uno de los diecisiete países megadiversos del mundo, lo cual implica potencial para que el país sea una reserva alimentaria y de medicinas a futuro.

En materia de propiedad intelectual, es la primera vez que el Perú ha logrado que países que tradicionalmente se oponen a reconocer cualquier vínculo entre la propiedad intelectual y los conocimientos tradicionales asociados a los recursos genéticos acepten dentro del Acuerdo, y en particular dentro del capítulo de propiedad intelectual, que existe un vínculo entre los derechos de propiedad intelectual y los conocimientos tradicionales y recursos genéticos asociados a estos, y además se comprometan a cooperar con el Perú en el cumplimiento de su legislación. Este logro es de suma importancia para el Perú, al ser un país con comunidades que detentan conocimientos ancestrales relacionados con los recursos genéticos que deben ser reconocidos y protegidos.

Es importante señalar también que el TPP cuenta con un capítulo de cooperación que promueve la colaboración entre sus miembros y la creación de capacidades en los países miembros menos adelantados. Las áreas de cooperación son amplias, pues cubren todos los temas cubiertos por el Tratado. Por ejemplo, el Perú podrá contar con cooperación que permita el desarrollo de su capital humano, pues se abre canales para propiciar el intercambio de experiencias en materia de educación, cultura y equidad de género.

Es necesario señalar que para el Perú ha sido menos complejo que para otros países del bloque TPP aceptar los estándares propuestos durante la negociación del Tratado. Ello se debe a que durante la etapa de negociaciones del TPP, el Perú ya contaba con 17 acuerdos comerciales en vigencia con las principales economías del mundo, y en muchos de esos acuerdos el país asumió compromisos similares a los que fueron propuestos posteriormente en la negociación del TPP.

Las obligaciones en el ámbito medioambiental y laboral son claros ejemplos de áreas en las cuales el Perú ya había implementado los compromisos del APC Perú - Estados Unidos, asumiendo estándares elevados. En consecuencia, la inclusión de estos temas en el TPP no solamente fue menos compleja de asumir para el Perú, sino que el país se beneficia en tanto que otros miembros del TPP, principalmente algunos países de la región asiática, deben elevar también sus estándares y de esta manera competirán con el Perú en igualdad de condiciones. 


\section{Recuadro 1 \\ Costos de no participar en el TPP}

Es importante conocer los beneficios del TPP, pero también los costos que podría enfrentar el Perú en caso de no aprobar y poner en vigencia el Tratado. Algunos de los costos serían los siguientes:

- Se enviaría una pésima señal al mercado, pues podría interpretarse como un cambio en la política de apertura comercial e integración al mundo que el Perú ha seguido en los últimos 25 años.

- En el plano internacional, se deterioraría la imagen del Perú como un país que persigue consistentemente su política de integrarse al mundo, y en especial a la región Asia-Pacífico. Los inversionistas extranjeros podrían retrasar o redirigir sus inversiones hacia países que se muestren más consecuentes con su política comercial y de atracción de inversiones.

- No participar en el TPP pone en riesgo la participación futura de Perú en la negociación de un acuerdo de libre comercio que integre a las 21 economías de APEC, conocido como Área de Libre Comercio del Asia Pacífico (FTAAP).

- El Perú perdería competitividad en el comercio internacional, frente a países como Chile y México, que se beneficiarían del mercado ampliado de 12 economías y la acumulación de origen, mientras que el Perú solamente se quedaría con acuerdos bilaterales.

- Se perdería la posibilidad de acceso con preferencias a cinco nuevos mercados y la posibilidad de mejorar el acceso a mercados con los países con los cuales el Perú ya tiene acuerdos comerciales vigentes.

- Se perdería el acceso a las cadenas globales de valor; es decir, la posibilidad de utilizar insumos de los otros 11 países del TPP para la fabricación de productos para que luego sean exportados en el marco del acuerdo, así como la posibilidad de integrar al Perú a la cadena productiva de la región TPP como abastecedor de insumos para otras industrias de la región.

- El Perú perdería la posibilidad de contar con disposiciones modernas sobre Medidas Sanitarias y Fitosanitarias, vinculantes y sujetas al mecanismo de solución de controversias del tratado.

- El Perú no formaría parte del primer acuerdo comercial que otorga a la PYME la más alta importancia. 


\section{Rompiendo mitos sobre el TPP}

Durante la etapa de negociación del TPP, así como luego de su suscripción, algunos grupos específicos, principalmente organizaciones no gubernamentales (ONG) han venido difundiendo aseveraciones sobre el Tratado que son incorrectas, tratando de direccionar la opinión pública hacia la oposición al Tratado.

$\mathrm{Al}$ respecto, es preciso recordar que los mismos temores se difundían en 2004 y 2005, durante la etapa de negociaciones del APC entre el Perú y los Estados Unidos. Las voces apocalípticas vaticinaban la desaparición del agro nacional, la elevación del precio de los medicamentos, así como el pago de millonarias compensaciones a inversionistas extranjeros. Luego de más de siete años de vigencia del APC Perú - Estados Unidos, la evidencia demuestra que nada de ello ocurrió, sino todo lo contrario: la agroexportación ha venido creciendo sostenidamente gracias a la apertura de mercados; los precios de las medicinas se redujeron en términos reales; y el Estado peruano ha enfrentado pocos arbitrajes internacionales, ganando la gran mayoría de ellos.

A continuación se analiza los mitos más importantes generados en torno a la participación del Perú en el TPP.

\subsection{Supuesto «secretismo» en la negociación}

Toda negociación internacional requiere de un nivel de confidencialidad adecuado para no afectar el proceso negociador en sí mismo. La difusión de propuestas que no son finales revelaría solamente intereses maximalistas de las partes, generando alarma innecesaria en grupos de interés y dificultando el proceso de negociación.

Ello sucede también en el ámbito privado. Si una persona pretende comprar un inmueble, no revela públicamente el precio máximo que está dispuesto a pagar, ni el vendedor revela el precio mínimo que está dispuesto a aceptar por dicho inmueble. La negociación conduce a un resultado mutuamente aceptable, que en la mayoría de casos se refleja en algún punto intermedio entre las posiciones maximalistas de negociación.

La negociación del TPP no ha sido en este aspecto distinta de otras negociaciones comerciales, ni ha sido una práctica aislada del Perú. Se cumplió con lo dispuesto en el artículo 15-A de la ley 27806, Ley de Transparencia y Acceso a la Información Pública, que dispone que los elementos de negociaciones internacionales, que de revelarse perjudicarían los procesos negociadores o alteraran los acuerdos adoptados, no serán públicos por lo menos en el curso de las mismas ${ }^{8}$.

\footnotetext{
8 Ley de Transparencia y Acceso a la Información Pública, Artículo 15-A.- Excepciones al ejercicio del derecho: Información Reservada. El derecho de acceso a la información pública no podrá ser ejercido respecto de la información
} 
Sin perjuicio de ello, el Mincetur llevó a cabo 29 reuniones informativas generales, antes y después de cada ronda de negociación. En dichas reuniones se convocó a gremios, organizaciones no gubernamentales y sector académico. Cada jefe de grupo de negociación expuso verbalmente los avances en la negociación de los capítulos a su cargo. Estas reuniones informativas generales fueron adicionales a las reuniones de coordinación permanentes que cada grupo de negociación llevó a cabo con los representantes acreditados de los sectores público y privado.

En forma adicional a estos informes, los miembros del Equipo Negociador, así como el jefe negociador, el viceministro de Comercio Exterior y la ministra de Comercio Exterior y Turismo participaron en exposiciones a los integrantes del sector privado y de la sociedad civil en general, según fue solicitado.

En el caso del Congreso de la República, se realizó presentaciones sobre el TPP en las comisiones de Comercio Exterior, Relaciones Exteriores, Salud y Protección al Consumidor.

\subsection{Mecanismo de Solución de Controversias Inversionista - Estado}

Un segundo mito que se ha tejido en torno a las negociaciones del TPP tiene relación con el Mecanismo de Solución de Controversias Inversionista - Estado, dispuesto en el capítulo sobre Inversión. Se ha afirmado que el Perú estaría cediendo su soberanía, por lo que empresas transnacionales podrían demandar al país ante tribunales internacionales y el Estado Peruano tendría que pagarles millonarias indemnizaciones.

$\mathrm{Al}$ respecto, debe señalarse que la solución de controversias entre inversionistas y el Estado, a través de los mecanismos de arbitraje internacional, es una práctica del Estado Peruano desde inicios de la década de 1990. Se adoptó como un mecanismo que brinda protección y certeza jurídica al inversionista, con el objetivo de generar confianza para invertir en el país. El TPP no cambia esta práctica.

El mecanismo de solución de controversias entre inversionistas y el Estado es consistente con lo establecido en el artículo 63 de la Constitución Política del Perú,

clasificada como reservada. En consecuencia la excepción comprende únicamente los siguientes supuestos:

[...]

2. Por razones de seguridad nacional y de eficacia de la acción externa del Estado, se considerará información clasificada en el ámbito de las relaciones externas del Estado, toda aquella cuya revelación originaria un riesgo a la seguridad e integridad territorial del Estado y la defensa nacional en el ámbito externo, al curso de las negociaciones internacionales y/o la subsistencia del sistema democrático. Estas excepciones son las siguientes: [...]

(a) Elementos de las negociaciones internacionales que de revelarse perjudicarían los procesos negociadores o alteraran los acuerdos adoptados, no serán públicos por lo menos en el curso de las mismas. 
que reconoce el arbitraje internacional como una vía válida para resolver controversias entre privados y el Estado?.

Con respecto a las supuestas indemnizaciones millonarias el Estado tendría que pagar a los inversionistas, es necesario revisar las estadísticas de fallos vinculados con arbitrajes internacionales en los que el Estado Peruano ha sido demandado. A pesar del gran número de Acuerdos Bilaterales de Inversión (BIT) y contratos que contienen cláusulas de arbitraje internacional, el Estado Peruano ha sido llevado a este tipo de arbitraje en contadas ocasiones. De los once casos resueltos, nueve han sido favorables al Estado Peruano o resueltos por mutuo acuerdo entre las Partes, mientras que únicamente dos de ellos resultaron favorables a los inversionistas y desfavorables para el Estado peruano. Sin embargo, en los dos únicos casos con fallos desfavorables, las compensaciones fueron mucho menores que las solicitadas por los inversionistas: en un caso la compensación fue de $50 \%$ y en otro $3 \%$ del monto solicitado ${ }^{10}$.

En el plano global, las estadísticas también son favorables a los Estados. Según un reporte de la UNCTAD al fin del año 2013, del total de casos de arbitraje internacional concluidos, aproximadamente $43 \%$ fue resuelto a favor de los Estados, $31 \%$ a favor de los inversionistas y $26 \%$ fue resuelto de común acuerdo. Por tanto, no puede afirmarse que los tribunales arbitrales internacionales de inversión están sesgados a favor de los inversionistas (UNCTAD IIA, 2015).

\subsection{Propiedad intelectual y precio de medicamentos}

Una de las principales críticas de los opositores al TPP está vinculada con una supuesta elevación de los precios de los medicamentos, a raíz de los compromisos asumidos en el capítulo de propiedad intelectual del Tratado, relacionados con patentes y protección de datos de prueba.

Cabe señalar que la protección de patentes y la protección de datos de prueba son conceptos distintos. Si bien ambas protecciones pueden traducirse finalmente en períodos de exclusividad en el mercado, la protección de patentes es una recompensa al esfuerzo que implica la actividad creativa humana (creación o perfeccionamiento de una invención), mientras que la protección de datos de prueba recompensa la inversión realizada por una empresa farmacéutica para llevar a cabo costosas pruebas en humanos para probar la seguridad y eficacia de un producto farmacéutico, lo cual es un requisito indispensable para la aprobación del medicamento para su comercialización.

\footnotetext{
9 Artículo 63.- [...] «El Estado y las demás personas de derecho público pueden someter las controversias derivadas de relación contractual a tribunales constituidos en virtud de tratados en vigor. Pueden también someterlas a arbitraje nacional o internacional, en la forma en que lo disponga la ley.»

10 Los laudos desfavorables al Estado peruano se dieron en los casos Duke Energy y Tza Yap Shum.
} 
Ambos mecanismos, tanto la protección de patentes como la de datos de prueba, promueven la innovación, que de otro modo no recibiría incentivo alguno.

En el caso de las patentes, el TPP mantiene el período de protección en veinte ańos contados a partir de la fecha de presentación de la solicitud. Este plazo se aplica en el Perú desde 1993, al haberse incorporado en la normativa de la Comunidad Andina (Decisión 344, sustituida por Decisión 486), de aplicación directa en el Perú, así como en el Acuerdo sobre los Aspectos de los Derechos de Propiedad Intelectual relacionados con el Comercio (ADPIC) de la Organización Mundial del Comercio $(\mathrm{OMC})^{11}$. No es cierto que el TPP establezca una ampliación del período de la protección por más allá de veinte años, como han sugerido grupos que critican al Tratado.

Es importante señalar que el TPP contiene un mecanismo de ajuste en el plazo de la patente ante dos supuestos: (i) retrasos irrazonables en el procesamiento de la patente por la autoridad de patentes, y (ii) retrasos irrazonables en el procesamiento del registro sanitario por la autoridad sanitaria. Este mecanismo dispone que las Partes deban implementar mecanismos para ajustar, al final del plazo de protección de la patente, aquellos retrasos irrazonables establecidos en los supuestos (i) y (ii) ${ }^{12}$.

El Perú cuenta con el mecanismo de compensación por retrasos irrazonables de la autoridad de patentes para todas las invenciones, excepto en el sector farmacéutico (Decisión 689 de la Comunidad Andina) y no cuenta con mecanismos para ajustar en plazos de patentes los retrasos de la autoridad sanitaria. En el TPP, se ha considerado la situación particular del Perú en el Anexo 18-D. Dicho Anexo reconoce que las Decisiones 486 y 689 de la Comunidad Andina restringen al Perú implementar estas disposiciones. Por tanto, el compromiso del Perú se circunscribe a realizar los mejores esfuerzos en la Comunidad Andina para obtener una dispensa y poder aplicar los ajustes descritos previamente. En caso el Perú demuestre que la Comunidad Andina niega la dispensa, el Perú solamente debe continuar asegurando que no discrimina en el otorgamiento de patentes. Es decir, en el supuesto de que la Comunidad Andina niegue la dispensa, se extingue la obligación de implementar los ajustes en el ámbito de las patentes de productos farmacéuticos.

\footnotetext{
11 La primera Decisión de la Comunidad Andina que adopta el plazo de 20 años de protección de para las patentes es la Decisión 344 - Régimen Común sobre Propiedad Industrial, de 21 de octubre de 1993, Sección IV. Este plazo se mantiene en la Decisión 486 - Régimen Común sobre Propiedad Industrial, Capítulo V, que sustituyó la Decisión 344 en setiembre de 2000.

12 Se considera retraso irrazonable de la autoridad otorgante de la patente, el retraso de más de cinco ańos contados a partir de la fecha de presentación de la solicitud, o de tres años desde la fecha en la que se haya hecho la solicitud de informe técnico.
} 
En el caso de la protección de datos de prueba, se dijo que el TPP establecería períodos de protección de doce años. Ello no es correcto. El TPP establece dos opciones: (i) proteger los datos de prueba por un período de 8 años; o (ii) proteger los datos de prueba por un período de 5 años, más otras medidas, como puede ser el período que se requiere para la tramitación del registro sanitario; y reconociendo que las condiciones comerciales también contribuyen a la efectiva protección comercial, de modo que se brinde un resultado comparable en el mercado. Por tanto, el Perú puede escoger entre las dos opciones de protección, siendo la de menor plazo la opción que implementaría el Perú.

Además el Perú logró en el TPP que el plazo de 5 años de protección de datos de prueba, tanto para medicamentos de síntesis química como biológica, empiece a contar desde la fecha del primer registro, que usualmente se da en Estados Unidos o la Unión Europea. Ello implica que los plazos se reducen en la práctica ${ }^{13}$.

Cabe señalar que el Perú ya cuenta en su legislación desde 2009 con protección de datos de prueba por cinco años para productos farmacéuticos de síntesis química, específicamente para las «nuevas entidades químicas». Esta protección se estableció como resultado de los acuerdos alcanzados en el APC Perú - Estados Unidos y fue posteriormente incorporada a la legislación nacional en $2009^{14}$.

Durante la negociación del APC entre Perú y los Estados Unidos, la protección de datos de prueba para productos farmacéuticos de origen biológico no estuvo siquiera en discusión, simplemente debido a que la negociación se llevó a cabo entre 2004 y 2005, años en los que el desarrollo de medicamentos biológicos, basados en la aplicación de procesos biotecnológicos, era incipiente o prácticamente nulo.

Otro mito que ha circulado a raíz de las negociaciones del TPP está relacionado con la supuesta suma de los períodos de protección de patentes y datos de prueba. Se dijo que ambos plazos, patente y datos de prueba, se sumarían, con lo cual la protección y los períodos de exclusividad podrían llegar hasta 32 años (20 de patente y 12 de datos de prueba). Ello es falso, las patentes y los datos de prueba son protecciones distintas que no se suman, sino coexisten en el tiempo. En la práctica, el período de protección de datos de prueba de hasta cinco años, se ubicará dentro del período de 20 años de protección de la patente. Por tanto, no se extienden los plazos de protección. El siguiente gráfico ilustra la coexistencia en el tiempo de ambas protecciones:

\footnotetext{
13 En el caso de las Nuevas Entidades Químicas, que ya se protegen hasta por cinco años en el Perú desde 2009, el plazo promedio de protección ha sido de tres años, debido a que el plazo empieza a contar desde la fecha del primer registro, que se da usualmente en el exterior.

14 Decreto legislativo 1072, modificado por ley 29316, y vigente desde el 1 de febrero de 2009, fecha de entrada en vigencia del APC Perú - Estados Unidos. El reglamento fue aprobado mediante Decreto supremo 002-2009-SA, publicado en el diario oficial El Peruano el 17 de enero de 2009.
} 
Gráfico 5. Coexistencia de la protección de patentes y datos de prueba

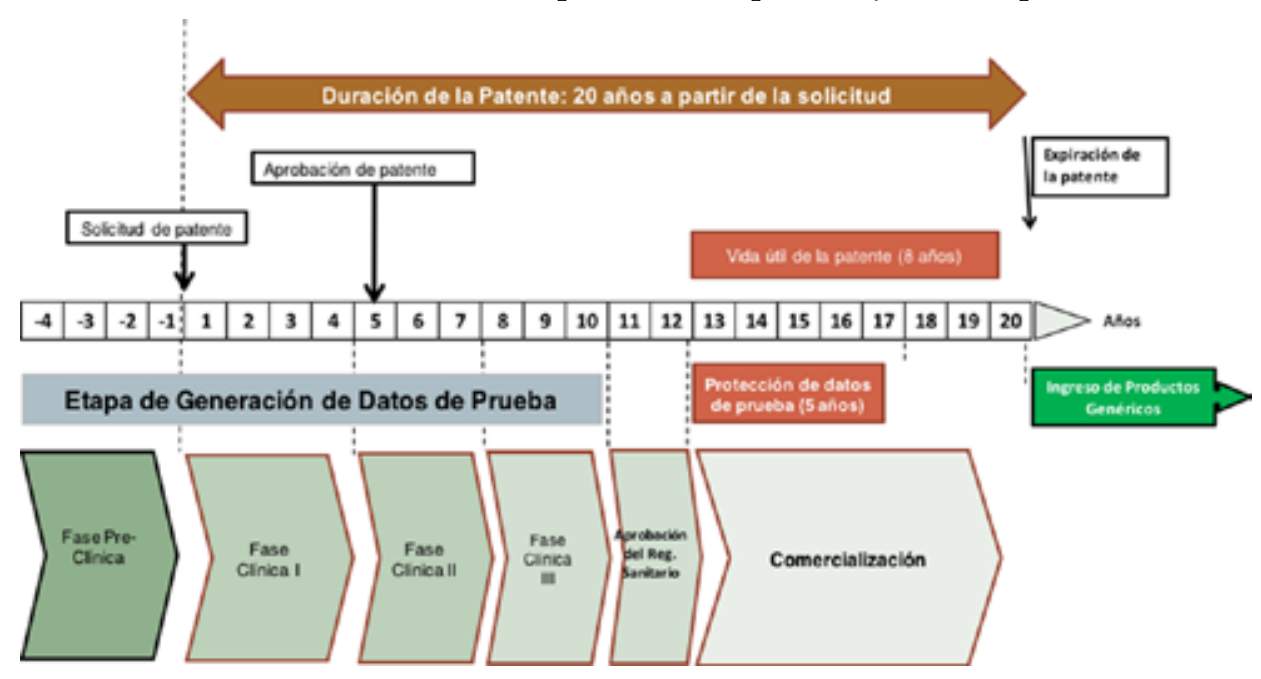

Fuente: Fedefarma. Elaboración: propia.

Algunos opositores al TPP también mencionan que se ha aceptado la protección de nuevos usos de medicamentos en el Tratado, con lo cual se podría extender los períodos de protección de patentes por muchos años más allá de los 20 años de protección que establece la legislación. Esa afirmación es falsa. El TPP otorga a sus miembros la libertad de patentar nuevos usos si es que ya lo vienen haciendo.

En el caso del Perú, la normativa andina no permite las patentes de nuevos usos. Por tanto, se tomó en consideración esta particularidad al negociar el TPP. El Tratado establece, en el artículo 18.37 (Materia Patentable) que las patentes deben estar disponibles para al menos uno de los siguientes: (i) nuevos usos de un producto conocido, (ii) nuevos métodos de usar un producto conocido, o (iii) nuevos procedimientos de uso de un producto conocido. Dado que en el Perú ya existe la protección de patentes para nuevos procedimientos, y que además se establece la posibilidad de no otorgar protección a procedimientos que reivindiquen usos, el Perú no cambiará su legislación en cuanto a la materia patentable ${ }^{15}$.

Es relevante señalar que los mismos temores que difunden actualmente los opositores al TPP, vinculados con una supuesta elevación de precios y escasez de medicamentos, se difundían durante las negociaciones del APC Perú - Estados Unidos, entre 2004 y 2005.

\footnotetext{
15 Para asegurar que el TPP no requiera al Perú patentar nuevos usos, se incluyó la siguiente frase en el párrafo 2 del artículo 18.37 en relación con las patentes de procedimiento: «Una Parte puede limitar dichos nuevos procedimientos a aquellos que no reivindiquen el uso del producto como tal».
} 
Sin embargo, la realidad ha demostrado que, luego de seis años de vigencia del APC bilateral, nada de ello ocurrió, sino todo lo contrario: la oferta de medicamentos se incrementó y abarató.

A continuación se muestra información que demuestra la evolución del mercado farmacéutico en el Perú, con posterioridad a la entrada en vigencia del APC Perú - Estados Unidos y luego de aplicación de los cinco años de protección de datos de prueba para productos farmacéuticos de origen químico (nuevas entidades químicas - NEQ):

- Según el INEI, mientras la inflación aumentó 3,7\% en promedio anual, los precios de los productos medicinales y farmacéuticos solamente crecieron a una tasa promedio de 3,5\% entre 2010 y 2015 . Ello quiere decir que en términos reales, luego de la entrada en vigencia del APC, los precios de los medicamentos se redujeron.

- Según IMShealth ${ }^{16}$ el panorama de precios del mercado farmacéutico peruano al año 2015 es el siguiente:

o El precio promedio real del mercado total de medicamentos decreció $19,8 \%$ con respecto a 2008 .

- El precio promedio real del mercado institucional de medicamentos decreció $46,8 \%$ con respecto a 2008 .

- El precio promedio real del mercado retail de medicamentos decreció $17,2 \%$ con respecto a 2008 .

o El precio promedio real de los 10 principales medicamentos oncológicos decreció $14,2 \%$ con respecto a 2008 .

o El precio promedio real de los 10 principales medicamentos contra el VIH decreció $28,8 \%$ con respecto a 2008 .

o El volumen del mercado total de medicamentos creció $33 \%$ entre 2008 y 2015.

- Según IMShealth, después de la entrada en vigencia del APC Perú - Estados Unidos, los medicamentos genéricos han alcanzado hasta $71 \%$ de las ventas en volumen en el mercado peruano, con un crecimiento promedio anual de $3 \%$.

Es importante notar también que de los miles de medicamentos que existen en el mercado peruano, solamente 26 NEQ cuentan con protección de datos de prueba vigente al 27 de abril de $2016^{17}$.

\footnotetext{
16 IMShealth es una compañía de los Estados Unidos que provee información, servicios y tecnología para la industria farmacéutica. Es líder mundial en provisión de datos e investigación de mercado para la industria farmacéutica.

17 Véase http://www.digemid.minsa.gob.pe/UpLoad/UpLoaded/PDF/ERPF/Datos_De_Prueba.pdf
} 


\subsection{Libertad de los usuarios en internet}

Otro de los mitos que ha circulado en torno al contenido del TPP señala que la libertad de expresión y de acceso a la información de los usuarios de internet se vería seriamente amenazada por el TPP.

Debe tomarse en cuenta que la legislación peruana, Ley sobre el Derecho de Autor, ya protege a los autores, intérpretes, productores de fonogramas por cualquier medio, creado o por crearse ${ }^{18}$. Ello, obviamente, incluye el entorno digital e internet.

$\mathrm{Al}$ respecto, es necesario aclarar que el TPP no contiene disposiciones que profundicen lo ya establecido en el APC entre Perú y los Estados Unidos en relación con las obligaciones de los proveedores de servicios de internet. El TPP no restringirá la libertad de los usuarios de internet, pues el capítulo de propiedad intelectual no se refiere a las libertades civiles de los usuarios de internet. Lo que se busca es la protección del derecho de autor y los derechos conexos en el entorno digital, tal como sucede en los medios físicos.

Con respecto a las responsabilidades de los proveedores de servicios de internet (PSI), las disposiciones del APC Perú - Estados Unidos aún no están implementadas en la legislación peruana. Estas disposiciones se refieren a las acciones que deben adoptar los PSI, tanto los que ofrecen acceso como los que ofrecen contenido, ante vulneraciones al derecho de autor, ante el requerimiento de una parte o una autoridad. Cabe precisar que bajo ninguna circunstancia se establece la obligación para el PSI de monitorear los contenidos que circulan en las redes. Una vez que se implementen dichas disposiciones del APC Perú - EE.UU., el TPP quedará automáticamente implementado en este aspecto.

\section{Suscripción, aprobación y entrada en vigencia}

A la fecha de elaboración de este artículo el TPP fue suscrito por el Estado peruano y se encuentra en la etapa de perfeccionamiento interno con el objetivo de lograr una pronta ratificación.

18 Decreto Legislativo 822, Ley de Derecho de Autor:

Artículo $30^{\circ}$.- El autor goza del derecho exclusivo de explotar su obra bajo cualquier forma o procedimiento, y de obtener por ello beneficios, salvo en los casos de excepción legal expresa.

Artículo $31^{\circ}$.- El derecho patrimonial comprende, especialmente, el derecho exclusivo de realizar, autorizar o prohibir: a) La reproducción de la obra por cualquier forma o procedimiento. b) La comunicación al público de la obra por cualquier medio.

[...]

f) Cualquier otra forma de utilización de la obra que no está contemplada en la ley como excepción al derecho patrimonial, siendo la lista que antecede meramente enunciativa y no taxativa. (Los resaltados son nuestros) 
La Constitución Política del Perú dispone de dos vías para el perfeccionamiento de los Tratados. La vía ejecutiva o la vía de aprobación por el Congreso de la República cuando se trate de Tratados que versen sobre derechos humanos, soberanía, dominio o integridad del Estado, defensa nacional, obligaciones financieras del Estado o aquellos que crean o suprimen tributos; los que exigen modificación o derogación de alguna ley o los que requieren medidas legislativas para su ejecución. En el caso del TPP, se trata de un Tratado que debe remitirse para aprobación del Congreso de la República, pues requiere medidas legislativas para su ejecución.

La medida legislativa de implementación del TPP más relevante es la vinculada con la protección de datos de prueba para medicamentos de origen biológico, aunque también deben implementarse otras disposiciones de la sección de observancia del capítulo de propiedad intelectual, como sanciones penales en caso de importación dolosa y de uso doméstico a escala comercial de etiquetas y embalajes, en los cuales una marca fue adherida sin autorización.

Una vez aprobado el TPP por el Congreso de la República, el Poder Ejecutivo emite un Decreto de Ratificación que es remitido al Depositario del Tratado, que en este caso es Nueva Zelanda.

La cláusula de entrada en vigencia del TPP establece que el Tratado entrará en vigencia en los siguientes supuestos:

a. A los 60 días siguientes a la fecha en la que todos los signatarios originales hayan notificado por escrito al Depositario la conclusión de sus procedimientos legales aplicables.

b. Si no ha ocurrido el supuesto señalado en a) en un plazo de dos años, el Tratado entrará en vigor a los 60 días siguientes a la expiración de este período si al menos seis de los signatarios originales, quienes en conjunto sumen al menos el 85 por ciento del producto interno bruto combinado de los signatarios originales en 2013, han notificado por escrito al Depositario la conclusión de sus procedimientos legales aplicables dentro de este plazo.

c. Si no han ocurrido los supuestos a) o b), el TPP entrará en vigor entrará en vigor a los 60 días siguientes a la fecha en la que al menos seis de los signatarios originales, quienes en conjunto sumen al menos el 85 por ciento del producto interno bruto combinado de los signatarios originales en 2013, hayan notificado por escrito al Depositario la conclusión de sus respectivos procedimientos legales aplicables.

Los signatarios originales que no se encuentren entre los países para los cuales el TPP entre en vigor de conformidad con los párrafos precedentes, deben someterse a la aprobación de la Comisión del TPP. Es por ello que es fundamental que el Perú se encuentre en el primer grupo de países para los cuales el Tratado entra en vigencia. 
De acuerdo con las cláusulas establecidas en el Tratado, y asumiendo celeridad en los procesos de perfeccionamiento interno de los miembros, se estima que el TPP entraría en vigencia en el primer semestre de 2018.

\section{Nuevos miembros y la futura Área de Libre Comercio del Asia Pacífico}

El texto del TPP dispone que el Tratado esté abierto preferentemente al ingreso de otras economías de APEC y luego a economías que no forman parte de este foro de cooperación. Por tanto, el TPP tiene un carácter evolutivo, cuyo espíritu es que el comercio internacional en la región del TPP se desarrolle bajo estándares y reglas similares, de modo que el comercio entre sus miembros fluya mejor y crezca con más fuerza.

Dado el tamaño de las economías que forman parte del TPP, este bloque ha concertado el interés de un importante número de economías de APEC, así como economías fuera de la región APEC. A la fecha han expresado interés en el TPP Corea del Sur, Tailandia, Taipéi, Indonesia y Filipinas, dentro del grupo de economías pertenecientes a APEC; así como Colombia y Costa Rica entre los que no son miembros de APEC.

Uno de los objetivos del TPP es continuar creciendo para convertirse en la base de la futura Área de Libre Comercio del Asia Pacífico (FTAAP). En este contexto, el TPP ya viene siendo analizado al interior de APEC, pues uno de los temas que el foro ha priorizado para 2016, el año en el que las reuniones de APEC se celebran en el Perú, es la entrega del Estudio de Factibilidad del FTAAP, el mismo que toma como base iniciativas como el TPP y el Regional Comprehensive Economic Partnership (RCEP) ${ }^{19}$. Es un reto para los miembros fundadores del TPP establecer los mecanismos e incentivos adecuados para que las economías interesadas puedan acceder a formar parte del TPP y, de esta manera, ampliar el bloque a futuro.

\footnotetext{
19 El RCEP es un acuerdo comercial que viene siendo negociado por 10 Estados de Asia y Oceanía: los miembros de la Asociación de Naciones del Sudeste Asiático - ASEAN (Brunéi, Myanmar, Cambodia, Indonesia, Laos, Malasia, Filipinas, Singapur, Tailandia y Vietnam) y seis Estados con los cuales ASEAN tiene Acuerdos de Libre Comercio (Australia, China, India, Japón, Corea del Sur y Nueva Zelanda).
} 


\section{Bibliografía}

Bhagwati, Jadish (1995). U.S. Trade Policy: The Infatuation with Free Trade Agreements. En Jagdish Bhagwati y Anne O. Krueger. The Dangerous Drift to Preferential Trade Agreements. Washinton D.C.: AEI Press.

Comunidad Andina (CAN) (1993). Decisión 344 - Régimen Común sobre Propiedad Industrial.

Comunidad Andina (CAN) (2000). Decisión 486 - Régimen Común sobre Propiedad Industrial.

Congreso de la República del Perú (1993). Constitución Política del Perú.

Congreso de la República del Perú (1996). Decreto Legislativo 822 - Ley de Derecho de Autor.

Congreso de la República del Perú (2008). Decreto Legislativo 1072 - Protección de datos de prueba u otros no divulgados de productos farmacéuticos.

Executive Office of the President of the United States (2008). 2008 Trade Policy Agenda and 2007 Annual Report of the President of the United States on the Trade Agreements Program. Washington D.C.

Mincetur (2015). 105 Preguntas y Respuestas sobre el Acuerdo de Asociación Transpacífico. Lima: Ministerio de Comercio Exterior y Turismo.

Pedri, Petri y Michael Plummer (2016). The Economic Effects of the Trans-Pacific Partnership: New Estimates. Washington D.C.: Peterson Institute for International Economics. Disponible en: <https://piie.com/publications/working-papers/economic-effectstrans-pacific-partnership-new-estimates $>$.

OEA. Organización de Estados Americanos (Sistema de Información sobre Comercio Exterior (SICE) de la Organización de Estados Americanos (OEA). Disponible en: $<$ www.sice.oas.org>.

Unctad IIA Issues Note (2015). Investor-State Dispute Settlement: Review of Developments in 2014. Número 2, mayo. Disponible en: <http://unctad.org/en/PublicationsLibrary/ webdiaepcb2015d2_en.pdf>.

World Bank (2016). Potential Macroeconomic Implications of the Trans-Pacific Partnership. En: Global Economic Prospects. Washington D.C.: World Bank.

Fecha de recepción: 30 de marzo de 2016 Fecha de aprobación: 29 de abril de 2016 